\title{
Apport de l'imagerie tomographique volumique à faisceau conique (cone beam) dans le diagnostic et la prise en charge des dens invaginatus
}

\section{RÉSUMÉ}
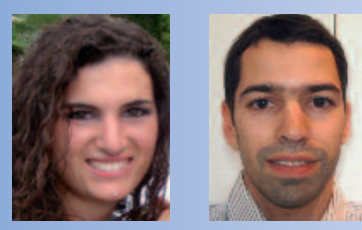

Esther AZOULAY

Docteur en chirurgie dentaire, CECSMO 1, Université Paris-Diderot,

Service d'orthodontie,

Hôpital Pitié-Salpétrière, Paris.

179 , rue Saint-Honoré,

75001 Paris.

Antoine BOUTON

Docteur en chirurgie dentaire,

CECSMO 1, Université René-Descartes,

Service d'orthodontie,

Hôpital Bretonneau, Paris.

179 , rue Saint-Honoré,

75001 Paris.

\section{Gérard PASQUET}

Médecin-Radiologue,

Cabinet de radiologie,

Échelle-Saint Honoré,

Paris.
L'invagination amélo-dentinaire, plus communément connue sous le terme de dens in dente, est une anomalie de développement de la dent qui affecte le plus souvent les incisives latérales maxillaires permanentes. Cette anomalie peut se présenter sous différentes formes, dont la connaissance permet une prise en charge adéquate. Cet article se propose de rappeler une classification utile et de montrer l'intérêt de l'imagerie tomographique volumique à faisceau conique (cone beam) comme outil diagnostique pour dépister et traiter ces anomalies morphologiques. 


\section{Introduction}

$>$

L'invagination amélo-dentinaire, plus

souvent connue sous le terme de dens in dente, est une anomalie de développement relativement rare qui résulte de l'invagination de la couche épithéliale avant le stade de minéralisation [1]. La fusion, union de la dentine et/ou de l'émail de deux dents séparées, peut conduire à ce type d'anomalie lorsqu'elle inclut le système canalaire.

L'étiologie des dens invaginatus est mal connue. Cependant, certaines théories tentent d'expliquer la survenue de cette anomalie : déformations de l'organe de l'émail lors du développement du germe sous la pression des germes

\section{Diagnostic clinique}

Le diagnostic clinique n'est pas simple. L'aspect clinique des dens invaginatus est très variable. La morphologie coronaire, normale la plupart du temps, peut trouver ses dimensions vestibulo-linguales augmentées, présenter un ergot cingulaire (talon cusp), adopter une forme particulière (conique...) [6-8]. Selon certains auteurs, la fréquente discrétion des signes cliniques des dens invaginatus est à l'origine d'un " sous-diagnos-

\section{Classification}

Un certain nombre de classifications ont été proposées $[5,6,12]$. La plus largement utilisée, probablement par sa simplicité d'application est celle de Oehlers [6] (fig. 1).

En se fondant sur la profondeur de l'invagination observée sur des radios, I'auteur distingue trois types de dens invaginatus: adjacents ; accélération localisée du développement de l'épithélium; infection ; trauma [2]. Sa prévalence varie entre $0,4 \%$ et $10 \%$, les incisives latérales maxillaires étant les plus atteintes [3,4].

Selon Patel et al., I'hétérogénéité des critères d'inclusion et d'exclusion, les populations étudiées et les méthodes de diagnostic des dens invaginatus expliquent la grande disparité des résultats retrouvés dans la littérature. Parmi les dents atteintes, $90 \%$ sont des incisives latérales maxillaires, 6,5\% seulement des dents postérieures [5]. De rares cas isolés concernent les incisives mandibulaires.

tic » de l'anomalie [5]. Pourtant, s'il est réalisé précocement, ce dernier permet de prévenir des complications pulpaires $[7,9,10]$. En effet, des défauts de structure tissulaire sont fréquemment retrouvés dans I'invagination $[4,7,11,12]$. Cette dernière, inaccessible aux matériels de brossage, est le point de départ de lésions carieuses, puis de pathologies pulpaires (nécrose, granulome ou kyste apico-dentaire) $[7,13,14]$.

- le type I : invagination minime confinée dans la couronne et ne s'étendant pas au-delà de la jonction amélo-cémentaire (JEC), la plus commune, $79 \%$ selon Ridell et al. [16] ;

- le type II est caractérisé par une invagination qui s'étend apicalement au-delà de la JEC dans la racine mais se termine dans un cul-de- 
sac, sans atteindre le ligament parodontal ou les tissus parodontaux, $15 \%$ selon Ridell et al. ;

- le type III, dans lequel l'invagination s'étend apicalement à travers la racine et crée un second foramen latéral (type IIla) ou apical (type IIlb) dans le ligament parodontal, $5 \%$ selon Ridell et al.

Cette classification est un outil précieux pour le praticien car elle oriente son choix thérapeu- tique. Les options de traitement sont nombreuses allant du simple scellement prophylactique du sillon au traitement canalaire complet par voie orthograde et/ou rétrograde $[6,15,16]$. L'extraction, autrefois recommandée [19], est aujourd'hui exceptionnelle [6]. Les critères décisionnels découlent d'une analyse radiologique poussée permettant l'identification du type d'invagination et la compréhension du réseau canalaire.

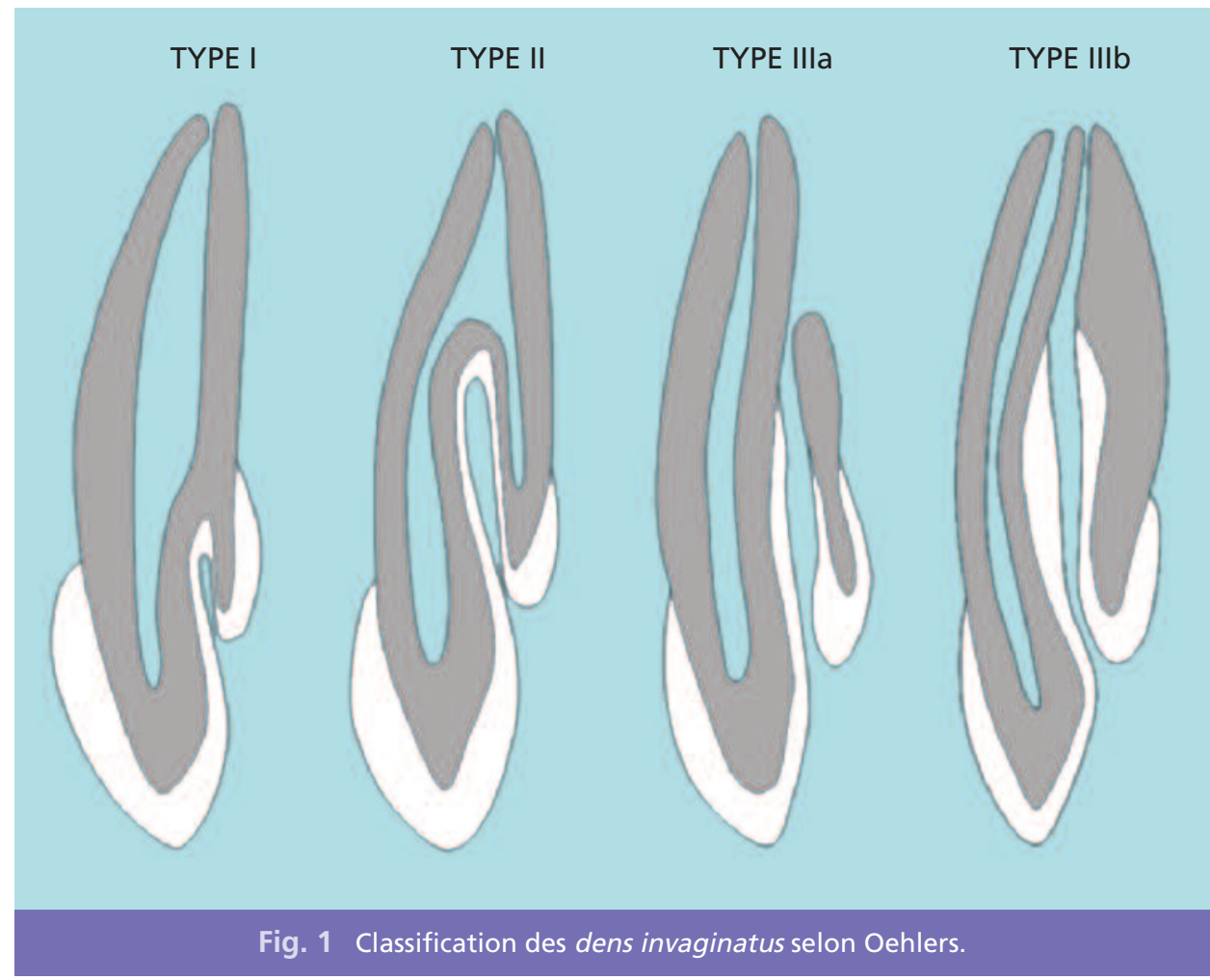

\section{Diagnostic radiologique}

Le diagnostic radiologique peut sembler aisé. En radiologie conventionnelle, l'image prend souvent l'aspect caractéristique d'une "flamme de bougie " avec l'impression de dent dans une dent [17].
Mais l'analyse radiologique doit permettre de déterminer la forme, l'étendue et les rapports de l'invagination avec le canal pulpaire ainsi que l'identification d'une éventuelle lésion [6]. 

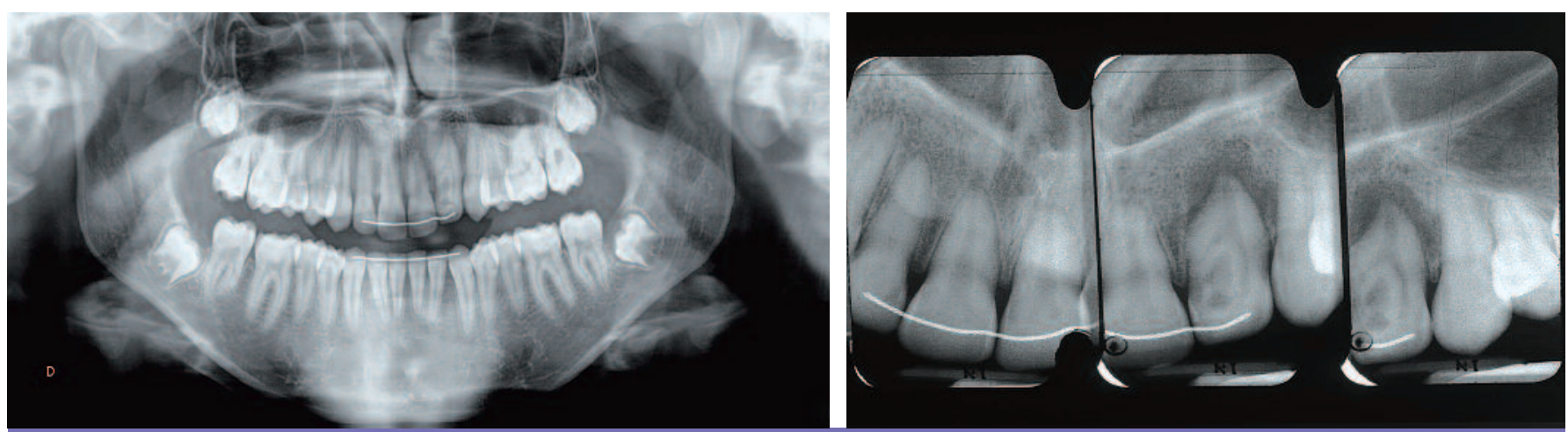

Fig. 2 a et b : Invagination amélo-dentinaire de type II sur 22, chez un adolescent de 14 ans. OPT et clichés retroalvéolaires.

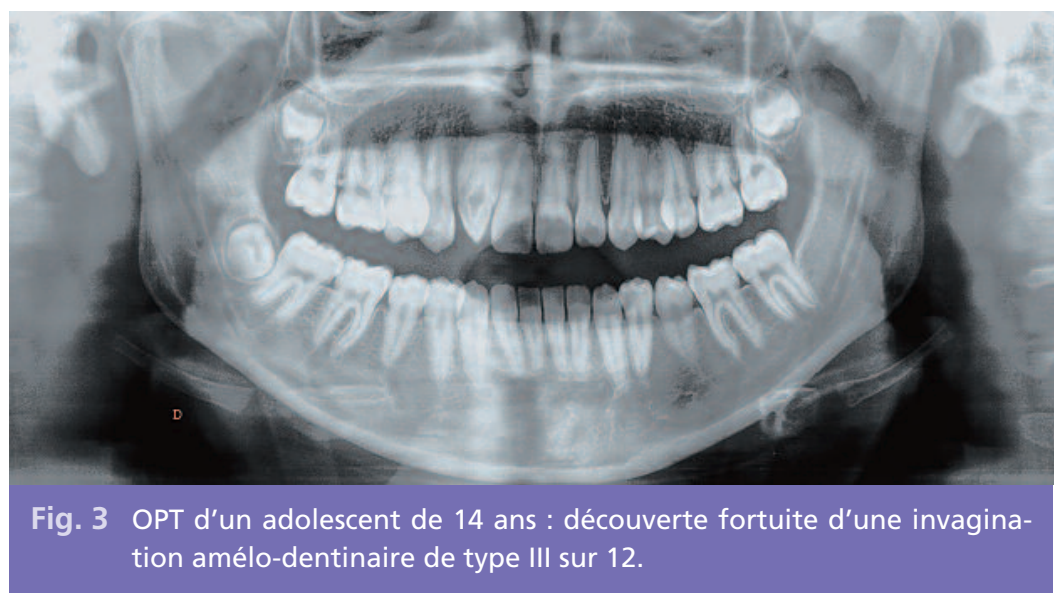

L'imagerie conventionnelle peut être suffisante mais impose souvent plusieurs clichés sous différentes angulations [5]. Les résultats dépendent beaucoup de l'expérience du praticien qui en fait I'analyse et en particulier de sa capacité à identifier un volume à partir d'une image en 2D. Elle est souvent insuffisante lorsqu'elle ne permet pas au clinicien d'apprécier précisément la complexité du réseau canalaire dans les trois sens de l'espace et rend aléatoire le diagnostic et la prise en charge de ce type d'anomalie (fig. 2 et 3) .

\section{Apport de l'imagerie sectionnelle}

Dernière-née de l'imagerie sectionnelle, la technique tomographique volumique numérisée à faisceau conique ou " cone beam » est dévolue à la seule imagerie dento-maxillaire.

Différente dans sa conception du scanner Rx, elle est économe en radiations, fiable en résul- tats et reconnue comme un mode d'imagerie substitutif au scanner [17].

D'après le rapport d'évaluation technologique publié par la HAS en 2009, " Tomographie volumique à faisceau conique de la face (cone beam computerized tomography: $C B C T$ ) », cette tech- 
nique sectionnelle autorise une imagerie de l'ensemble du complexe maxillo-facial ou une exploration complète ou limitée des structures maxillo-mandibulaire et dento-alvéolaire.

Elle trouve ainsi son indication dans différents domaines de l'odontologie : implantologie, chirurgie, endodontie, parodontologie, orthodontie... Elle constitue un outil diagnostique de choix et notamment ici dans le diagnostic et la prise en charge des dens invaginatus.

On trouve sur le marché plusieurs types d'appareils dont les applications sont différentes. Les appareils « petit champ » offrent une résolution spatiale optimale $(80 \mu)$ très appréciable en endodontie. Les appareils « grand champ » autorisent une exploration totale des maxillaires (ATM compris) comme des explorations plus sectorielles en réduisant le champ (et la dose d'irradiation). La tendance est à la polyvalence en cherchant à développer des appareils de champ moyen capables d'accomplir toutes les tâches [18].

Les coupes axiales, sagittales et coronales ci-dessous (fig. 4) illustrent bien l'anatomie atypique d'une invagination amélo-dentinaire touchant I'incisive latérale maxillaire droite encore immature chez un enfant de 9 ans.
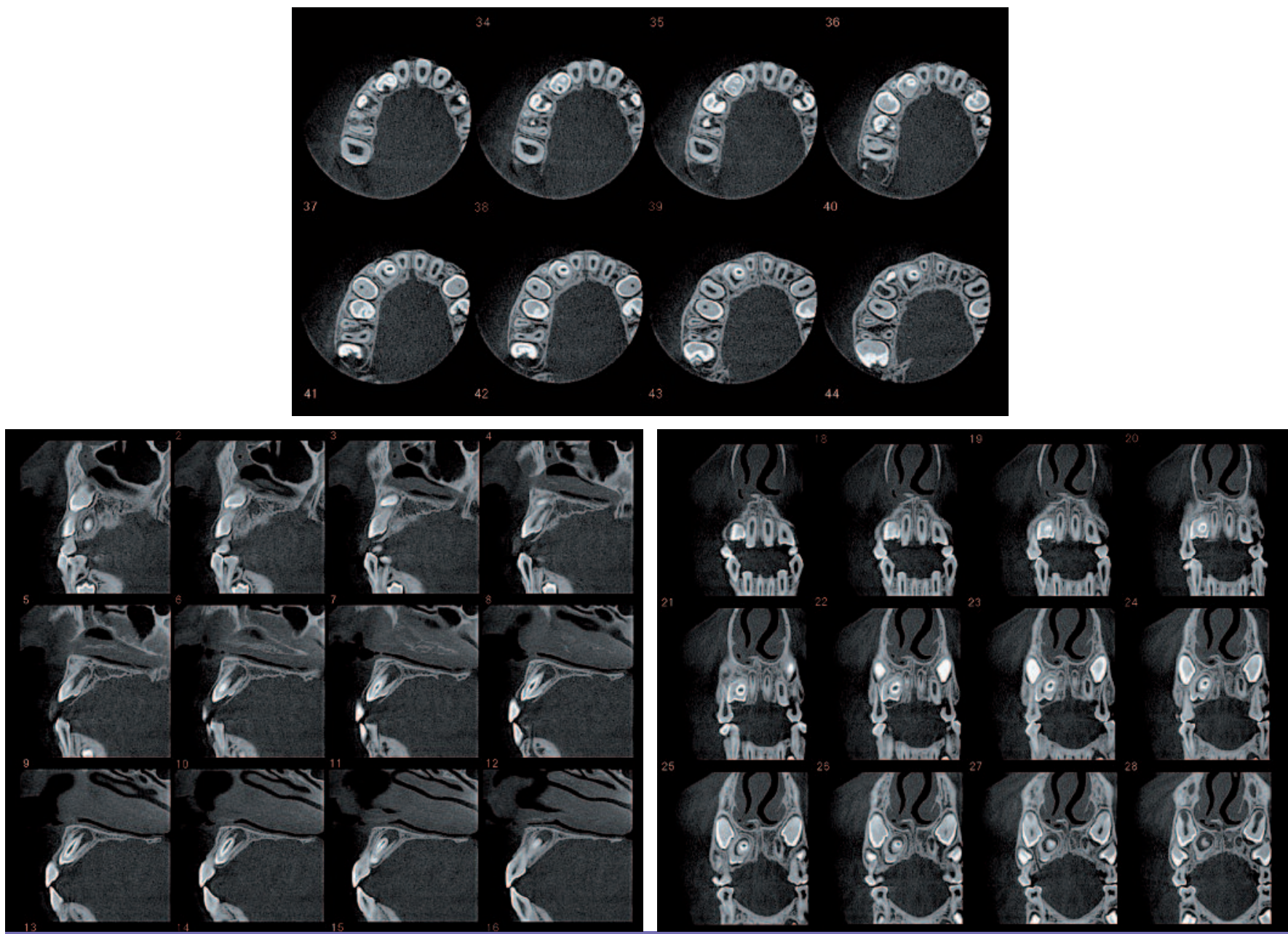

Fig. 4 a à c Diagnostic d'une invagination amélo-dentinaire chez un enfant de 9 ans : coupes axiales, sagittales et coronales d'un examen tomographique volumique. 


\section{Conclusion}

L'invagination amélo-dentinaire, anomalie du développement dentaire résultant d'une augmentation ou d'une invagination de la papille dentaire, constitue un cas particulier où l'originalité anatomique vient accroître les difficultés de nettoyage, désinfection et obturation de l'ensemble du réseau canalaire, lorsqu'un traitement endodontique est à envisager.
Pour mener à bien son traitement, le praticien désireux de prendre en charge ce type d'anomalie doit faire appel à des méthodes diagnostiques performantes.

Dans cette optique, l'imagerie sectionnelle moderne "cone beam», peu irradiante, lui apporte l'aide précieuse qui vient pallier les insuffisances d'un protocole d'imagerie conventionnelle en deux dimensions trop imprécis.

\section{Bibliographie}

1. Carvalho-Sousa B, Almeida-Gomes $\mathrm{F}$, Gominho LF, Albuquerque DS. Endodontic treatment of a periradicular lesion on an invaginated type III mandibular lateral incisor. J Dent Res 2009;20(2):243-5.

2. Patel S.

The use of cone beam computed tomography in the conservative management of dens invaginatus: a case report. Int Endod J 2010 Aug;43(8):707-13.

3. Durack C, Patel S. The use of cone beam computed tomography in the management of dens invaginatus affecting a strategic tooth in a patient affected by hypodontia: a case report. Int Endod J 2011 May;44(5):474-83.

4. Reddy YP, Karpagavinayagam K,
Subbarao CV.

Management of

dens invaginatus diagnosed

by spiral computed

tomography: a case report.

$J$ Endod

2008 Sep;34(9):1138-42.

5. Alani A, Bishop K.

Dens invaginatus.

Part 1: classification, prevalence and aetiology. Int Endod J

2008 Dec;41(12):1123-36.

6. Bishop K, Alani A.

Dens invaginatus.

Part 2: clinical,

radiographic features and management options. Int Endod J

2008 Dec;41(12):1137-54.

7. Oehlers FA.

Dens invaginatus (dilated composite odontome).

I. Variations of the invagination process and associated anterior crown forms.

Oral Surg Oral Med

Oral Pathol 1957 Nov;10(11):

1204-18 contd.
8. Khabbaz MG,

Konstantaki MN, Sykaras SN.

Dens invaginatus

in a mandibular lateral

incisor.

Int Endod J

1995 Nov;28(6):303-5.

9. Oelhers FA.

Dens invaginatus (dilated composite odontome).

II. Associated posterior crown forms and pathogenesis. Oral Surg Oral Med Oral Pathol 1957 Dec;10(12):1302-16.

10. Rushton MA. Invaginated teeth (dens in dente): contents of the invagination. Oral Surg Oral Med Oral Pathol 1958 Dec;11(12):1378-87.

11. Kramer IR. The pathology of pulp death in non-carious maxillary incisors with minor palatal invaginations.

Proc R Soc Med 1953 Jul;:46(7):503-5. 
12. Vincent-Townend J.

Dens invaginatus.

J Dent

1974 Nov;2(6):234-8.

13. Beynon AD.

Developing

dens invaginatus

(dens in dente).

A quantitative microradiographic study and a reconsideration of the histogenesis of this condition.

Br Dent J 1982 Oct;153(7):255-60.

14. De Smit A, Jansen HW, Dermaut L.

An histological investigation of invaginated human incisors.
J Biol Buccale

1984 Sep;12(3):201-9.

15. Keleş A, Cakici F.

Endodontic treatment of a maxillary lateral incisor with vital pulp, periradicular lesion and type III dens invaginatus: a case report. Int Endod J 2010 Jul;43(7):608-14.

16. Kristoffersen $\varnothing, \mathrm{Nag} \mathrm{OH}$, Fristad I.

Dens invaginatus and treatment options based on a classification system: report of a type II invagination. Int Endod J 2008 Aug;41(8):702-9.
17. Cavezian R., Pasquet G., Bel G., Baller G. Imagerie dento-maxillaire : approche radio-clinique. (3e éd.). Issy-les-Moulineaux : Elsevier Masson, 2006:32-6.

18. Cavezian R., Pasquet G. Cone beam : imagerie diagnostique en odontostomatologie. Principes, résultats et perspectives. Issy-les-Moulineaux : Elsevier Masson, sept. 2011.

19. Dechaume M.

Précis de Stomatologie $4^{\text {th }}$ ed.

Paris: Masson and Cie, 1966:567.

\section{SUMMARY}

\section{Contribution of}

Cone Beam Computerized Tomography in the diagnosis and the care of dens invaginatus

Esther AZOULAY,

Antoine BOUTON,

Gérard PASQUET

\section{Keywords \\ - dens invaginatus \\ - cone beam}

Dens invaginatus (DI), commonly known as dens in dente, is a developmental malformation of teeth that most commonly affects permanent maxillary incisor teeth. DI can present in a variety of forms, knowledge of which can help in diagnosis and treatment. The goal of this article is to remind a useful classification and to show the computerized tomography (Cone Beam) as a tool to diagnose and treat those morphological anomalies. 